\title{
Information Theory and Wigner Crystallization: A Model Perspective
}

\author{
David Thompson ${ }^{1}$, James S. M. Anderson ${ }^{2}$, and Kalidas Sen ${ }^{3}$ \\ ${ }^{1}$ Chemical Computing Group \\ ${ }^{2}$ Universidad Nacional Autónoma de México \\ ${ }^{3}$ University of Hyderabad School of Chemistry
}

August 28, 2020

\begin{abstract}
Accurate restricted Hartree-Fock (RHF) wave-functions are used to investigate information theoretic properties of the model problem of two interacting electrons confined within an infinite spherical potential of radius R. Benchmark quality calculations are performed to characterise this system via a range of information measures as a function of the tunable parameter $\mathrm{R}$, across the full electron correlation regime (low to high correlation; small $\mathrm{R}$ to large $\mathrm{R}$ ). Both the Shannon information entropy and a statistical complexity measure provide quantitative insight regarding the onset of the formation of a 'Wigner molecule' state for this system.
\end{abstract}

\section{Hosted file}

Thompson_Anderson_Sen_IJQC_2e_RHF_Information.docx available at https://authorea.com/users/ 354473/articles/478148-information-theory-and-wigner-crystallization-a-model-perspective 